\title{
Light Leakage of Multidomain Vertical Alignment LCDs Using a Colorimetric Model in the Dark State
}

\author{
Chuen-Lin Tien $\mathbb{D}^{1,2}$ Rong-Ji Lin, ${ }^{2}$ and Shang-Min Yeh $\mathbb{D}^{3}$ \\ ${ }^{1}$ Department of Electrical Engineering, Feng Chia University, Taichung 40724, Taiwan \\ ${ }^{2}$ Ph.D. Program of Electrical and Communications Engineering, Feng Chia University, Taichung 40724, Taiwan \\ ${ }^{3}$ Department of Optometry, Central Taiwan University of Science and Technology, Taichung 40601, Taiwan \\ Correspondence should be addressed to Shang-Min Yeh; optom.yap@gmail.com
}

Received 30 March 2018; Accepted 30 April 2018; Published 3 June 2018

Academic Editor: Jia-De Lin

Copyright (C) 2018 Chuen-Lin Tien et al. This is an open access article distributed under the Creative Commons Attribution License, which permits unrestricted use, distribution, and reproduction in any medium, provided the original work is properly cited.

Light leakage from liquid crystal displays in the dark state is relatively larger and leads to a degraded contrast ratio and color shift. This work describes a novel colorimetric model based on the Muller matrix that includes depolarization of light propagating through liquid crystal molecules, polarizers, and color filters. In this proposed model, the chromaticity can be estimated in the bump and no-bump regions of an LCD. We indicate that the difference between simulation and measurement of chromaticity is about 0.01 . Light leakage in the bump region is three times that in no-bump region in the dark state.

\section{Introduction}

Many wide viewing-angle LCDs have been investigated and produced. In-Plane Switching (IPS) [1, 2] and Multidomain Vertical Alignment (MVA) [3-5] are widely used for highend LCD products. The MVA LCD has a superior contrast ratio at normal viewing directions, wide viewing angle, and poor color dispersion. However, the light leakages in the dark state and color shift at an inclined angle have not yet been improved. Therefore, enhancing the contrast ratio and eliminating the light leakage in the dark state are important goals [6, 7]. Many conditions result in the light leakage such as light scattering from liquid crystals and the color filter, misalignment of crossed polarizers, and retardation of phase from liquid crystals $[8,9]$. Polarization light scattering from the liquid crystals due to thermally excited orientation-based fluctuations of liquid crystal directors and light depolarization from the color filter due to pigment scattering have to be discussed $[10,11]$. Investigating the colorimetric and photometric characteristics of LCDs, which include their major optical components, such as crossed polarizers, liquid crystals, color filters, and backlight modules, is interesting [12-14].

In this colorimetric model based on the Muller matrix, the transmission spectrum of each component is imported and the light scattering from liquid crystals is ignored. The chromaticity coordinates can be estimated by measuring the degree of depolarization of the polarizer and the color filter. The error of chromaticity can be analyzed by introducing three backlight units and color resists. Finally, this work presents simulation and measurement results for a LCD module in the dark state, which demonstrate that a minimum difference exists in chromaticity, and light leakage in the bump region is three times larger than in no-bump region.

\section{Analyzing Light Leakage in the Dark State}

Figure 1 shows some LCD parts-the polarizer, analyzer, liquid crystal, and color filter. Two major parts in pixel design exist for an MVA: one is the bump region and the other is the no-bump region. In the bump region, due to the shape of the bump, the orientation of liquid crystal molecules is affected by the boundary condition. When linear polarized light passes through the bump region, phase retardation results in nonlinear polarization light. Finally, light leakage can exist in the bump region. Furthermore, transmittance in the bump region also influences chromaticity of an LCD. We should be concerned with calculating individually the optical performance of a pixel in the bump and no-bump region. Figure 2 presents vertical images of the subpixel 


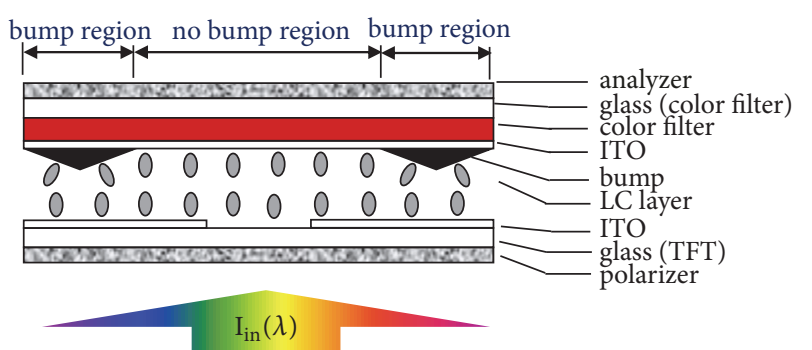

FIGURE 1: Scheme of MVA liquid crystal module.

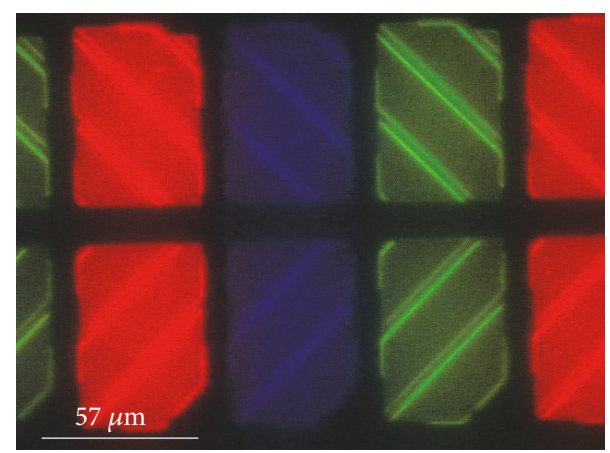

FIGURE 2: The vertical view images of subpixel color filter in dark state.

color filter at a normal direction in the dark state. The blue, green, and red lights are generated by the color filter with the existence of different color pigments. The oblique lines in each grid (subpixel) indicate the bump region, which is brighter than the no-bump region in the dark state due to light leakage. Light leakage can reduce colorimetric and photometric performance of liquid crystal displays in the dark state.

\section{Colorimetric Calculation by Matrix Method}

Depolarization occurs with the reduction in degree of polarization when light scatters through optical elements such as the polarizer or color filter. In (1), the light emanating out of the LCD through the polarizer, analyzer, liquid crystals, and color filter can be described using a matrix of optics.

$$
P\left(\theta_{1}\right) D(d) P\left(\theta_{2}\right)\left(\begin{array}{c}
I \\
Q \\
U \\
V
\end{array}\right)=\left(\begin{array}{c}
I^{\prime} \\
Q^{\prime} \\
U^{\prime} \\
V^{\prime}
\end{array}\right),
$$

where $P(\theta)$ is the Muller matrix of polarizer; $\theta$ is the direction of the transmit axis and the subscript numbers of $\theta$ are the polarizer and analyzer, respectively; $D(d)$ is the Muller matrix of the depolarizer (including the polarizer and color filter); $d$ is the degree of depolarization. Notably, $I, Q, U$, and $V$ are known as stoke parameters of a quasimonochromatic plane wave that represents total intensity, horizontally or vertically polarized state, linear polarization along directions at angle $\varphi= \pm 45^{\circ}$ to the $x$-axis, and the right or left circularly polarized state, respectively. Superscripts of stoke parameters describe the conditions of light propagating through the LCD module. Values of $Q, U$, and $V$ vanish in the unpolarized state.

We assume the polarizer and analyzer comprise an ideal polarizer. The Muller matrix of the polarizer can be presented as follows:

$$
p(\theta)=\frac{1}{2}\left(\begin{array}{cccc}
1 & \cos 2 \theta & \sin 2 \theta & 0 \\
\cos 2 \theta & \frac{1+\cos 4 \theta}{2} & \frac{\sin 4 \theta}{2} & 0 \\
\sin 2 \theta & \frac{\sin 4 \theta}{2} & \frac{1-\cos 4 \theta}{2} & 0 \\
0 & 0 & 0 & 0
\end{array}\right) \text {, }
$$

where the Muller matrix in different directions of the transmit axes can be obtained by changing the value of $\theta$.

Equation (3) derives the contrast ratio determined by the intensity of outgoing light with parallel polarizers $\left(\theta_{1}=\theta_{2}=\right.$ 0 ) divided by that with crossed polarizers $\left(\theta_{1}=0, \theta_{2}=\pi / 2\right)$ in the dark state.

$$
\mathrm{CR}_{\mathrm{PDP}}=\frac{I_{/ /}^{\prime}}{I_{\perp}^{\prime}}=\frac{(2-d) / 4}{d / 4}=\frac{2-d}{d},
$$

where $I^{\prime} / /$ and $I_{\perp}^{\prime}$ are the total intensity of outgoing light with the polarization of polarizers parallel or orthogonally intersected with each other, respectively.

The colorimetric and photometric performance of LCDs depends on wavelength. That is, all mechanisms of light leakage should be determined in wavelength-related terms. Thus, considering the transmittance of color filters and polarizers, the degree of depolarization is function of wavelength. The $d(\lambda)$ can be estimated by (3) after measuring the contrast ratio. The intensity of outgoing light from the bump and nobump regions is as follows:

$$
\begin{aligned}
I_{\mathrm{BUMP}}^{\prime}(\lambda)= & \frac{d(\lambda)}{4} \times T_{\mathrm{CF}} \times T_{\mathrm{BUMP}} \times T_{\mathrm{LC}_{-} \mathrm{BUMP}} \\
& \times T_{\mathrm{PF}} \times T_{\mathrm{TFT}} \times I_{\mathrm{in}}(\lambda) \\
I_{\text {No-BUMP }}^{\prime}(\lambda)= & \frac{d(\lambda)}{4} \times T_{\mathrm{CF}} \times T_{\mathrm{LC} \_ \text {No-BUMP }} \times T_{\mathrm{PF}} \\
& \times T_{\mathrm{TFT}} \times I_{\mathrm{in}}(\lambda),
\end{aligned}
$$

where $T$ is transmittance of optical components, CF is the color filter, BUMP is the region with a bump, LC_BUMP is the region of the liquid crystal (LC) with a bump, PF is the polarizer or analyzer, TFT is thin-film transistors, and $I_{\text {in }}(\lambda)$ is light source spectrum.

To determine the chromaticity of the dark state, $X_{\text {bump }}$, $Y_{\text {bump }}$, and $Z_{\text {bump }}$ tristimulus values of the Commission 
International de 1'Éclairage (CIE) $X Y Z$ color space through the bump region can be written in the following form [14]:

$$
\begin{aligned}
& X_{\text {bump }}=k \int \frac{d(\lambda)}{4} \times T_{\mathrm{CF}}(\lambda) \times T_{\mathrm{BUMP}}(\lambda) \\
& \quad \times T_{\text {LC_BUMP }}(\lambda) \times T_{\mathrm{PF}}(\lambda) \times T_{\mathrm{TFT}}(\lambda) \times \bar{x}(\lambda) \\
& \quad \times I_{\text {in }}(\lambda) d \lambda \\
& Y_{\text {bump }}=k \int \frac{d(\lambda)}{4} \times T_{\mathrm{CF}}(\lambda) \times T_{\mathrm{BUMP}}(\lambda) \\
& \quad \times T_{\text {LC_BUMP }}(\lambda) \times T_{\mathrm{PF}}(\lambda) \times T_{\mathrm{TFT}}(\lambda) \times \bar{y}(\lambda) \\
& \quad \times I_{\text {in }}(\lambda) d \lambda \\
& Z_{\text {bump }}=k \int \frac{d(\lambda)}{4} \times T_{\mathrm{CF}}(\lambda) \times T_{\mathrm{BUMP}}(\lambda) \\
& \quad \times T_{\mathrm{LC} \_ \text {BUMP }}(\lambda) \times T_{\mathrm{PF}}(\lambda) \times T_{\mathrm{TFT}}(\lambda) \times \bar{z}(\lambda) \\
& \quad \times I_{\text {in }}(\lambda) d \lambda,
\end{aligned}
$$

where $\bar{x}(\lambda), \bar{y}(\lambda)$, and $\bar{z}(\lambda)$ are the tristimulus values of the monochromatic stimuli and $k$ is a constant.

Furthermore, the tristimulus values of light through the no-bump region $X_{\text {no_bump }}, Y_{\text {no_bump }}$, and $Z_{\text {no_bump }}$ can be written as follows:

$$
\begin{aligned}
& X_{\text {No_bump }}=k \int \frac{d(\lambda)}{4} \times T_{\mathrm{CF}}(\lambda) \times T_{\text {LC_NoBUMP }}(\lambda) \\
& \quad \times T_{\mathrm{PF}}(\lambda) \times T_{\mathrm{TFT}}(\lambda) \times \bar{x}(\lambda) \times I_{\text {in }}(\lambda) d \lambda \\
& Y_{\text {No_bump }}=k \int \frac{d(\lambda)}{4} \times T_{\mathrm{CF}}(\lambda) \times T_{\text {LC_NoBUMP }}(\lambda) \\
& \quad \times T_{\mathrm{PF}}(\lambda) \times T_{\mathrm{TFT}}(\lambda) \times \bar{y}(\lambda) \times I_{\text {in }}(\lambda) d \lambda \\
& Z_{\text {No_bump }}=k \int \frac{d(\lambda)}{4} \times T_{\mathrm{CF}}(\lambda) \times T_{\mathrm{LC} \_ \text {NoBUMP }}(\lambda) \\
& \quad \times T_{\mathrm{PF}}(\lambda) \times T_{\mathrm{TFT}}(\lambda) \times \bar{z}(\lambda) \times I_{\text {in }}(\lambda) d \lambda
\end{aligned}
$$

When analyzing the colorimetric and photometric performance of LCDs, the $X, Y$, and $Z$ values are difficult to interpret as the bump and no-bump regions coexist. The chromaticity coordinates $x, y$, and $z$ have been developed and established by the Commission International de 1'Éclairage (CIE). Equation (7) shows the relationship between chromaticity and tristimulus; as $x+y+z=1$, describing chromaticity of the stimulus using chromaticity coordinates $(x, y)$ is reasonable. To calculate optical performance of a pixel, the bump and no-bump regions should be investigated individually. This work sets ratio $A$, which is the bump area divided by the aperture area of a pixel, and the chromaticity $(x, y)$ of module can be derived in the dark state using $(8)$.

$$
\begin{aligned}
& x=\frac{X}{X+Y+Z}, \\
& y=\frac{Y}{X+Y+Z}, \\
& z=\frac{Z}{X+Y+Z} \quad X_{\text {bump }} \times A+X_{\text {No_bump }} \times(1-A) \\
& x=\frac{Y_{\text {bump }} \times A+Y_{\text {No_bump }} \times(1-A)}{\left(X_{\text {bump }}+Y_{\text {bump }}+Z_{\text {bump }}\right) \times A+\left(X_{\text {No_bump }}+Y_{\text {No_bump }}+Z_{\text {No_bump }}\right) \times(1-A)} \\
& y=\frac{\left(X_{\text {bump }}+Y_{\text {bump }}+Z_{\text {bump }}\right) \times A+\left(X_{\text {No_bump }}+Y_{\text {No_bump }}+Z_{\text {No_bump }}\right) \times(1-A)}{(1-A)}
\end{aligned}
$$

\section{Measurement}

Figure 3 shows the measurement system, in which a pair of polarizers are superimposed onto both sides of the prepared sample; the polarization of polarizers is parallel or orthogonally intersected. The light source is a backlight unit with cold cathode fluorescent lamp (CCFL) (AUO, 59.23M01.002), which can provide luminance of $>7500 \mathrm{~cd} / \mathrm{m}^{2}$. The light from the backlight unit passes through the polarizer (Nitto, NPFSEG1224DU), color resists (JSR Co., R874, G894, B877), analyzer, and spectrometer (Minolta, CS-1000), sequentially. The color filter is composed of three different color resists (R874, G894, B877 of R, G, and B, respectively). The distance between the first polarizer and backlight unit is $2 \mathrm{~cm}$ such that the incident angle is $<10^{\circ}$, and the distance between the two polarizers is $2 \mathrm{~cm}$. The colorimetric model can be implemented when the degree of depolarization $d(\lambda)$ is measured. This measurement must identify the contrast ratio that is equal to the luminance of the light passing through the parallel polarizers divided by that crossing the polarizers.

\section{Simulation Results of Light Leakage in the Dark State}

The degree of depolarization of RGB color resists is measured without addressing the transmittance of the black matrix. The contrast ratio of the color filter can be measured using various RGB color resists. If the liquid crystal and TFT layers are absent, the spectrum of outgoing light depends 


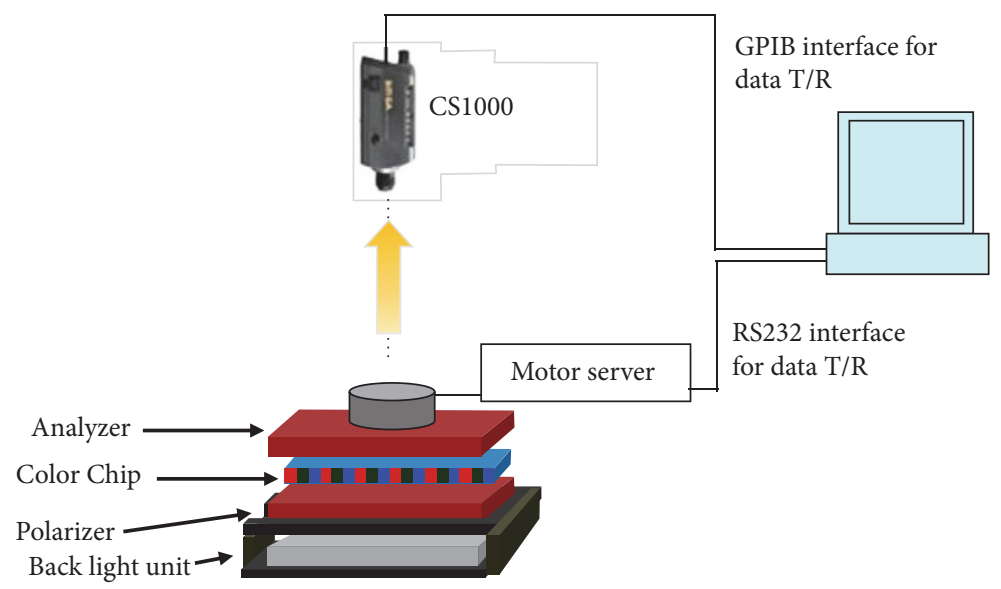

FIGURE 3: Auto-experimental system of measurement for contrast ratio of color resists.

\begin{tabular}{|c|c|c|c|c|c|c|c|c|c|c|c|c|}
\hline \multirow{3}{*}{ BUMP } & \multicolumn{3}{|c|}{ RED } & \multicolumn{3}{|c|}{ GREEN } & \multicolumn{3}{|c|}{ BLUE } & \multicolumn{3}{|c|}{ White } \\
\hline & $\mathrm{x}$ & $y$ & $\mathrm{Y}$ & $\mathrm{x}$ & $y$ & $\mathrm{Y}$ & $\mathrm{x}$ & $\mathrm{y}$ & $\mathrm{Y}$ & $\mathrm{x}$ & $\mathrm{y}$ & $\mathrm{Y}$ \\
\hline & 0.5711 & \begin{tabular}{|l}
0.3782 \\
\end{tabular} & 0.0093 & 0.2487 & 0.5269 & \begin{tabular}{|l}
0.0258 \\
\end{tabular} & \begin{tabular}{|l}
0.1454 \\
\end{tabular} & 0.0563 & 0.0040 & \begin{tabular}{|l}
0.253 \\
\end{tabular} & \begin{tabular}{|l|l|} 
& 0.271 \\
\end{tabular} & 0.013 \\
\hline \multirow{3}{*}{ No_BUMP } & \multicolumn{3}{|c|}{ RED } & \multicolumn{3}{|c|}{ GREEN } & \multicolumn{3}{|c|}{ BLUE } & \multicolumn{3}{|c|}{ White } \\
\hline & $\mathrm{x}$ & $y$ & $\mathrm{Y}$ & $\mathrm{x}$ & $y$ & $\mathrm{Y}$ & $\mathrm{x}$ & $\mathrm{y}$ & $\mathrm{Y}$ & $\mathrm{x}$ & $\mathrm{y}$ & $\mathrm{Y}$ \\
\hline & $\begin{array}{l}0.5015 \\
\end{array}$ & 0.4216 & 0.0054 & 0.1798 & 0.3679 & 0.0061 & 0.1552 & 0.0431 & \begin{tabular}{|l}
0.0009 \\
\end{tabular} & 0.251 & 0.245 & 0.004 \\
\hline
\end{tabular}

FIGURE 4: Simulated result of chromaticity and photometry $(Y)$ in bump and no-bump region.

on the transmission spectrum of the color filter, degree of depolarization, and spectrum of the backlight unit in (4). In the colorimetric model, the values of $Y_{\text {parallel }}$ and $Y_{\text {cross }}$ are 14.05 and 0.002765 , respectively, under the white point condition. This model indicates that the value of contrast ratio is 5110 for a color filter. The contrast ratio of modeling is close to the actual measurement result of 5500. It is a rapid way of evaluating optical performance of color filters when developing new color resists.

The colorimetric and photometric performance $(x, y, Y)$ can be found (Figure 4) by considering the transmittance of the TFT, bump, and LC using (5) and (6). Without addressing the ratio of the bump area over a pixel area in this model, light leakage in the bump region is three times larger than nobump region. By improving the contrast ratio of LCDs, light leakage caused by LC retardation in the bump region can be reduced.

Figure 5 shows the output spectra of measurement (solid line) and simulations (dotted line) of an LCD module in the dark state. The spectra peaks at about $440 \mathrm{~nm}, 550 \mathrm{~nm}$, and $610 \mathrm{~nm}$, represent the R, G, and B colors, respectively. The $485 \mathrm{~nm}$ and $585 \mathrm{~nm}$ peaks generated overlap transmission spectra between the B and G color filters. The $710 \mathrm{~nm}$ peak is from the backlight emitter, which has high transmittance in the $\mathrm{R}$ region. Figure 5 demonstrates that measurement and simulation results are similar. Figure 6 shows the measurement and simulation results for the colorimetric model in the dark state and bright state with three panel positions. The solid and dotted lines represent the measurement and simulation results, respectively. The points in the upperright (down-left) side of figure are the bright state (dark

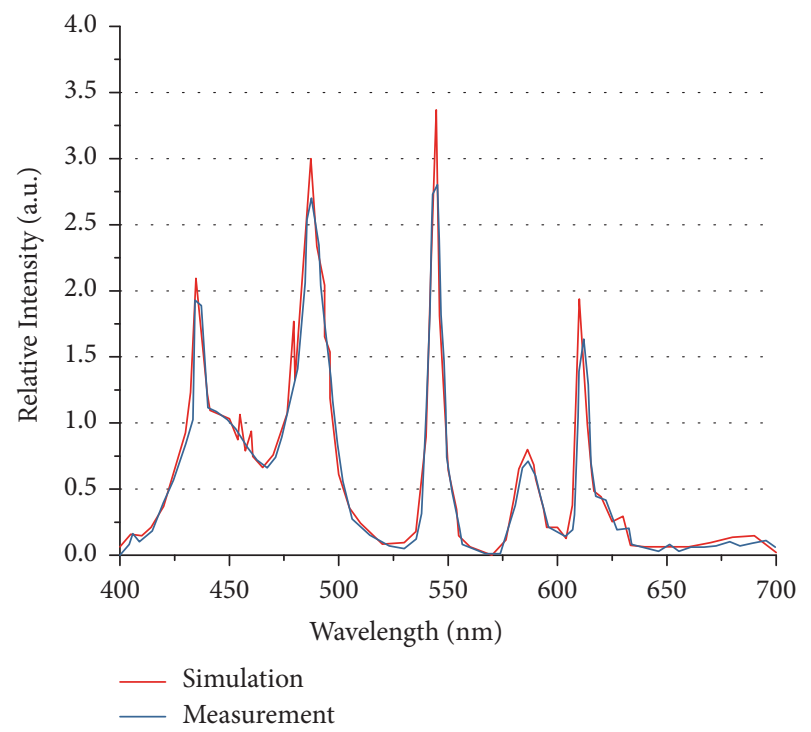

FIGURE 5: Output spectra of measurement and simulation of LCD module in the dark state.

state). In the bright state, the error of $(x, y)$ chromaticity is about 0.003; in the dark state, chromaticity error is about 0.01 . The large error in the dark state has two causes. One is the degree of depolarization in this colorimetric model. When the polarization of polarizers is crossed, light intensity is quite low, which results in fluctuations in the degree of depolarization in terms of wavelength. The other cause is error caused by manufacturing variation, such as polarizer 


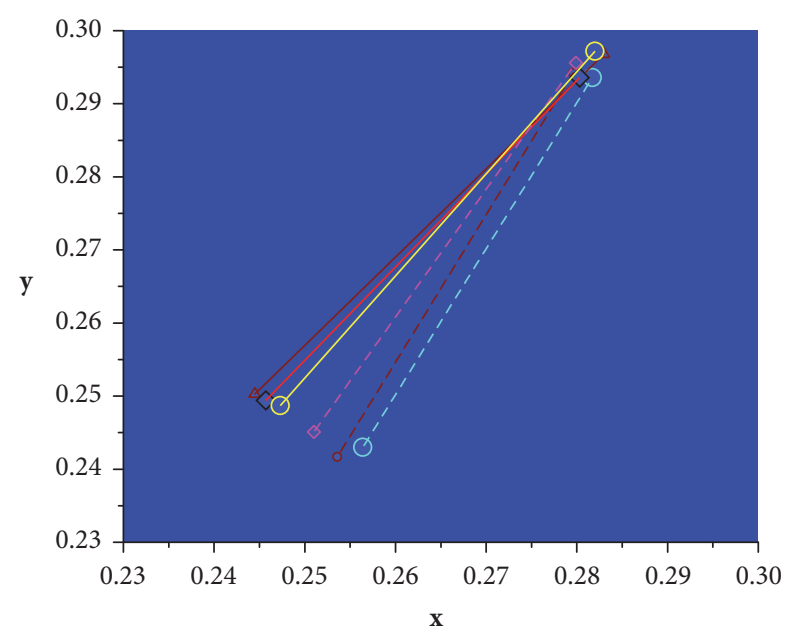

FIGURE 6: The chromaticity coordinates of measured and simulated results in the dark state and bright state.

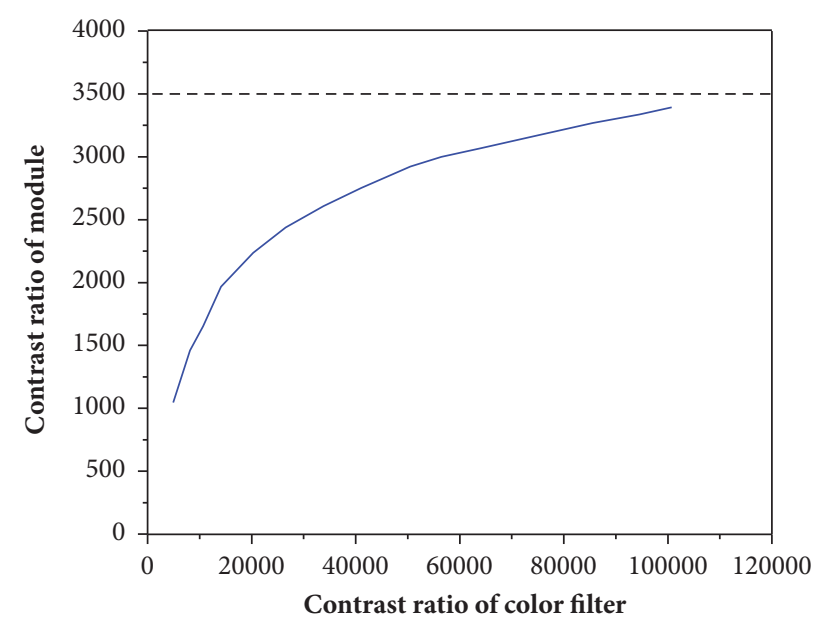

FIGURE 7: Simulation result for the contrast ratio performance of color filter and module.

misalignment or space in the cell gap variation. The low light intensity can be improved using a relatively brighter continuous light source. When degree of depolarization noise is minimized, estimating chromaticity of an LCD in the dark state is possible by measuring the degree of depolarization of color resists.

The contrast ratio is defined by $Y_{\text {bright }} / Y_{\text {dark }}$. Not only is the depolarization of the color filter an influential factor, but the light leakage in the bump area has an important role when calculating the contrast ratio. This model can evaluate the excellence of the contrast ratio of the LCD module when only improvements to the contrast ratio of the color filter are considered. Figure 7 shows the contrast ratio of the color filter, which increases with the contrast ratio of the LCD module when the extreme ratio of the LCD model is assumed to be about 3500. Actually, for a dispersion-type color filter, improving the contrast ratio of the color filter by $200 \%$ is difficult. Finally, the primary cause of contrast ratio deterioration of LCDs is light leakage from the bump region.

\section{Conclusions}

This work investigated the colorimetric and photometric properties of LCDs using a novel colorimetric model. This work demonstrates that the chromaticity coordinates of an LCD can be simulated accurately, and the proposed model can be utilized to optimize color LCDs with different optical components (excluding liquid crystals). Most importantly, this work thoroughly analyzes the colorimetric properties of LCDs and, in doing so, demonstrates that properly optimized LCDs use different backlights and color resists. Finally, the depolarization and retardation effects can be reduced to improve contrast ratio performance in an MVA-type LCD module.

\section{Data Availability}

The data used to support the findings of this study are available from the corresponding author upon request.

\section{Conflicts of Interest}

The authors declare that they have no conflicts of interest.

\section{Acknowledgments}

This research was supported by the Ministry of Science and Technology (MOST) of Taiwan under Grants MOST 1062221-E-035-072-MY2.

\section{References}

[1] S. Aratani, H. Klausmann, M. Oh-e et al., "Complete suppression of color shift in in-plane switching mode liquid crystal displays with a multidomain structure obtained by unidirectional rubbing," in Proceedings of the 1997 Pacific Rim Conference on Lasers and Electro-Optics, CLEO/Pacific Rim, p. 93, July 1997.

[2] C.-H. Chen, F.-C. Lin, and H.-P. D. Shieh, "A Field Sequential Color LCD Based on Color Fields Arrangement for Color Breakup and Flicker Reduction," Journal of Display Technology, vol. 5, no. 1, pp. 34-39, 2009.

[3] H. Chen, R. Zhu, M.-C. Li, S.-L. Lee, and S.-T. Wu, "Pixelby-pixel local dimming for high-dynamic-range liquid crystal displays," Optics Express, vol. 25, no. 3, pp. 1973-1984, 2017.

[4] H. Chen, G. Tan, M.-C. Li, S.-L. Lee, and S.-T. Wu, "Depolarization effect in liquid crystal displays," Optics Express, vol. 25, no. 10, pp. 11315-11328, 2017.

[5] S. H. Hong, Y. H. Jeong, H. Y. Kim, H. M. Cho, W. G. Lee, and S. H. Lee, "Electro-optic characteristics of 4-domain vertical alignment nematic liquid crystal display with interdigital electrode," Journal of Applied Physics, vol. 87, no. 12, pp. 82598263, 2000.

[6] T.-H. Yoon, G.-D. Lee, and J. C. Kim, "Nontwist quarter-wave liquid-crystal cell for a high-contrast reflective display," Optics Expresss, vol. 25, no. 20, pp. 1547-1549, 2000.

[7] Q. Hong, T. X. Wu, X. Zhu, R. Lu, and S.-T. Wu, "Designs of wide-view and broadband circular polarizers," Optics Express, vol. 13, no. 20, pp. 8318-8331, 2005.

[8] K. Okumoto, T. Tsuchiya, K. Yonemura et al., "A novel simulation method in In-Plane switching mode panel with 
considering light scattering behavior," in Proceedings of the 2015 SID International Symposium, pp. 1555-1558, usa.

[9] R. Lu, X. Zhu, S. Wu, Q. Hong, and T. Wu, "Ultrawide-View Liquid Crystal Displays," Journal of Display Technology, vol. 1, no. 1, pp. 3-14, 2005.

[10] M. Yoneya, Y. Utsumi, and Y. Umeda, "Depolarized light scattering from liquid crystals as a factor for black level light leakage in liquid-crystal displays," Journal of Applied Physics, vol. 98, no. 1, Article ID 016106, 2005.

[11] H. Chen, F. Peng, Z. Luo et al., "High performance liquid crystal displays with a low dielectric constant material," Optical Materials Express, vol. 4, no. 11, pp. 2262-2273, 2014.

[12] J. L. Pezzaniti, S. C. McClain, R. A. Chipman, and S.-Y. Lu, "Depolarization in liquid-crystal televisions," Optics Expresss, vol. 18, no. 23, pp. 2071-2073, 1993.

[13] M. Bass, Handbook of Optics, vol. 3, 1995.

[14] G. Wyszecki and W. S. Stiles, Color Science. Concepts and Methods, Quantitative Data and Formulae, 2000. 

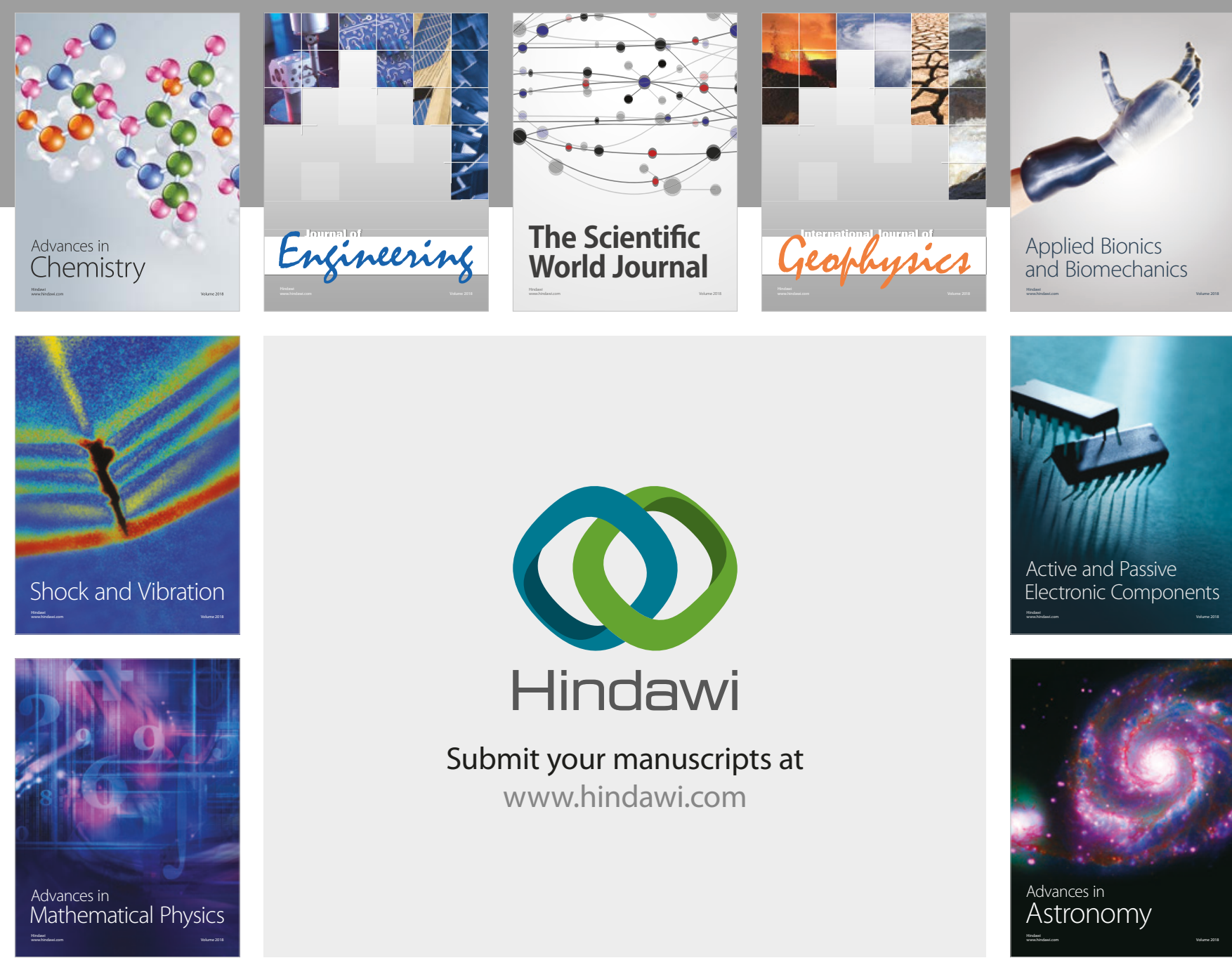

Submit your manuscripts at

www.hindawi.com

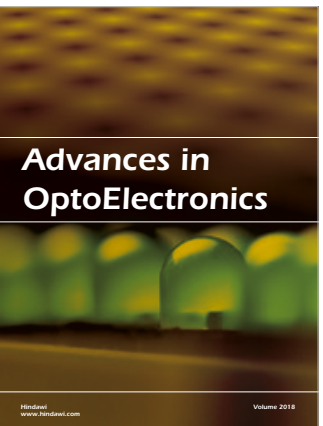

\section{Rotcting Machinery}
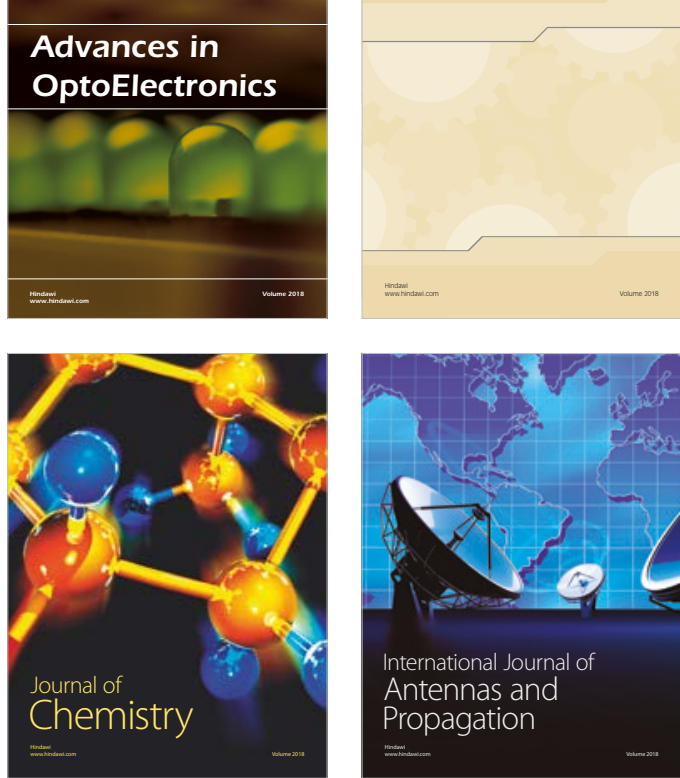

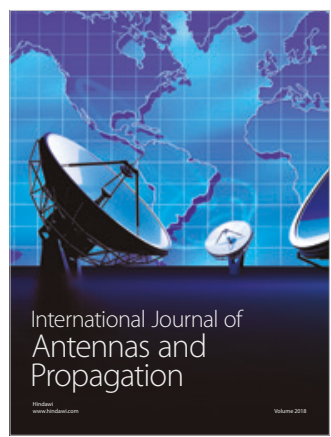

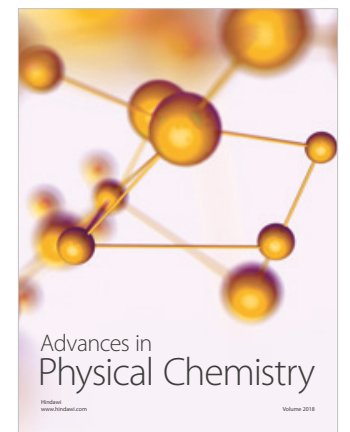

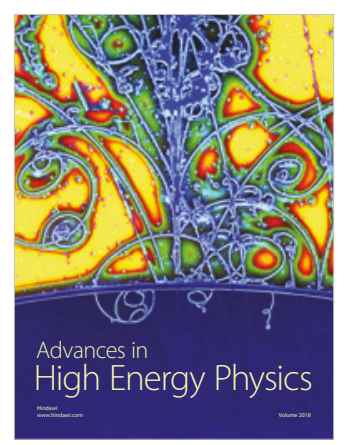

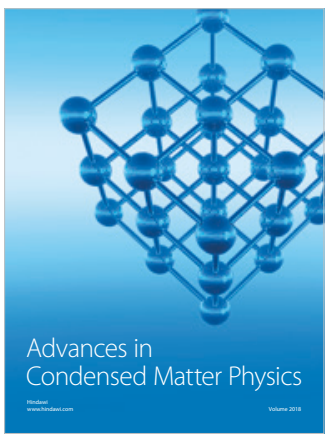

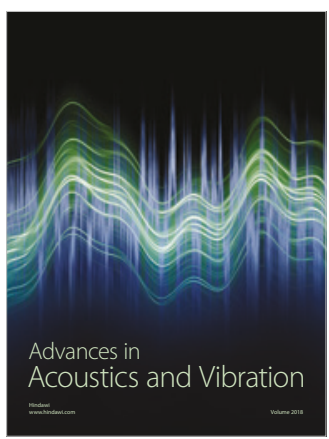

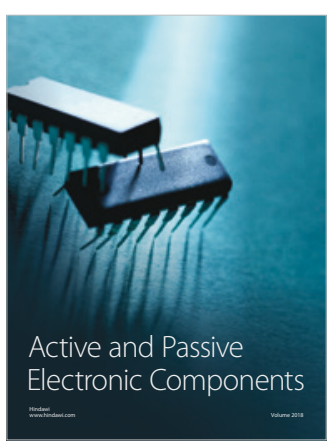
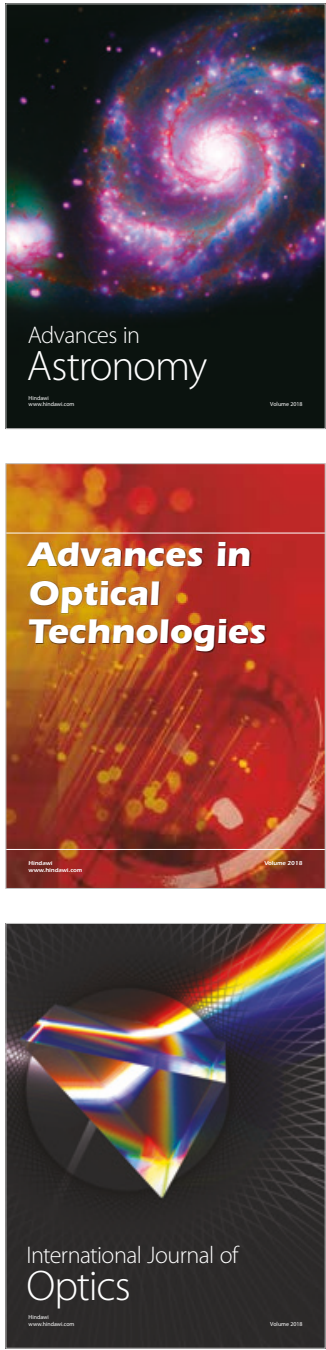